\title{
Texture Recognition: Evaluating Force, Vibrotactile and Real Feedback
}

\author{
Jonatan Martínez ${ }^{1}$, Arturo S. García ${ }^{2}$, Diego Martínez ${ }^{1}$, \\ José P. Molina ${ }^{1}$, and Pascual González ${ }^{1}$ \\ ${ }^{1}$ LoUISE Research Group, \\ Universidad de Castilla-La Mancha \\ ${ }^{2}$ Symbia IT, Parque Científico y Tecnológico de Albacete \\ 02071 Albacete, Spain \\ jonatan.martinez@uclm.es, arturo@symbiait.com, \\ \{diegomp1982, jpmolina, pgonzalez\} ddsi.uclm.es
}

\begin{abstract}
A force-feedback Phantom device, a custom-built vibrotactile dataglove, and embossed paper sheets are compared to detect different textures. Two types of patterns are used, one formed by different geometrical shapes, and the other with different grooves width. Evaluation shows that the vibrotactile dataglove performs better in the detection of textures where the frequency of tactile stimuli varies, and it is even useful to detect more complex textures.
\end{abstract}

Keywords: force feedback, vibrotactile; textures, dataglove.

\section{Introduction}

Haptic feedback is of vital importance in manipulative and exploration tasks of the daily life, as stated in [1]. Unfortunately, many interfaces for virtual environments do not provide this kind of feedback. One of the tasks that have been carried out to verify the effectiveness of this technology is the identification of materials and textures. Minsky et al. [2] used a joystick to experiment with force feedback, using a depth map texture. Some other authors attempted to optimize these systems when used to distinguish different materials [3] [4]. Tactile feedback can be used as a complement to force feedback, but it is also useful by its own, and it is often used even to replace it. In particular, vibrotactile feedback uses vibrations to transmit sensations through the skin, which plays an essential role in the way the different textures are detected [5]. There are tasks in which vibrations can increase the performance, reducing the response times or minimizing the forces used. Small modified speakers were used in [6] and, in a similar experiment, [7] used vibrotactile tactors to discriminate materials of different stiffness. A different actuator, the vibrator motor, has reduced bandwidth but is integrated in multiple devices, such as gaming peripherals [8], mobile phones, and datagloves [9].

In this context of texture identification, Kyung et al. [10] describes an experiment that compares force, tactile and vibrotactile feedback technologies. It was considered of interest to continue this experiment and use the same basis in order to have a reference to compare the results with. In the proposed study, some changes have been 
introduced, the vibrator is integrated in a dataglove and located directly on the fingertip, one of the most sensitive areas of the body [11], the control algorithm has been be optimized to reduce its latency, and some paper patterns have been introduced so that a real model can be considered in the analysis of the results. Next section will detail the patterns and the haptic feedback methods used.

\section{Description of the Haptic Feedback Methods}

The comparison of the three haptic feedback methods was performed using a discrimination task involving the identification of textures that followed some predefined patterns. Two of the three groups of patterns shown in [10] were used in this experiment. In the first group, each pattern is composed of four times the same geometric shape, while in the second each pattern is formed by horizontal lines with different spacing between them. These patterns were converted into tangible images where the black areas are $1 \mathrm{~mm}$ deeper than white ones (Fig 1 -left). In order to distinguish them, the user must perform scanning movements with each of the haptic approaches considered:

a) Force feedback - Sensable Phantom. With this device [12] and the H3DAPI library [13], textures were represented in a virtual box with patterns embossed in its upper side, using a depth map where the gray level determined the relative displacement. An X3D model was created for each pattern, and the Ruspini algorithm was selected for the haptic rendering.

b) Vibrotactile feedback. The second method is a dataglove capable of providing vibrotactile feedback that was developed in our laboratory [14]. This device has a small vibrating actuator, located on the index finger, whose frequency can be varied by the pulse width modulation generated on a microcontroller. The finger was tracked by a PhaseSpace system, attaching one LED on top of the fingertip (Fig. 1 -right).

c) Direct stimulation. In this case, the user moves her finger directly on the texture. It is the ideal tactile feedback, because the latency is zero, and the bandwidth and resolution are only limited by the sensitivity of the skin. The patterns are built using transparent paper $1 \mathrm{~mm}$ thick, removing the black areas to create zones of palpable depression. Patterns were placed on a table beneath another larger table that hid it form the user.
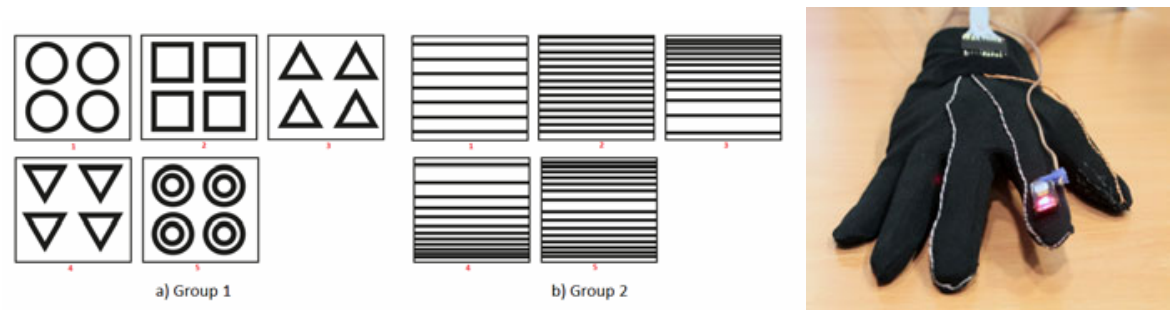

Fig. 1. Groups of patterns used (left) and custom-made dataglove (right) 


\section{Experiment Design}

Twelve different users (4 women and 8 men) were requested to distinguish the patterns of both groups using the three aforementioned methods. After each trial the users were informed about the correct answer. To prevent that the order could influence the results, the sequence was determined by the Latin square method. After each test, users were asked to rate the time they spent learning to detect patterns, the difficulty to distinguish them in an advanced phase, and the comfort of each device.
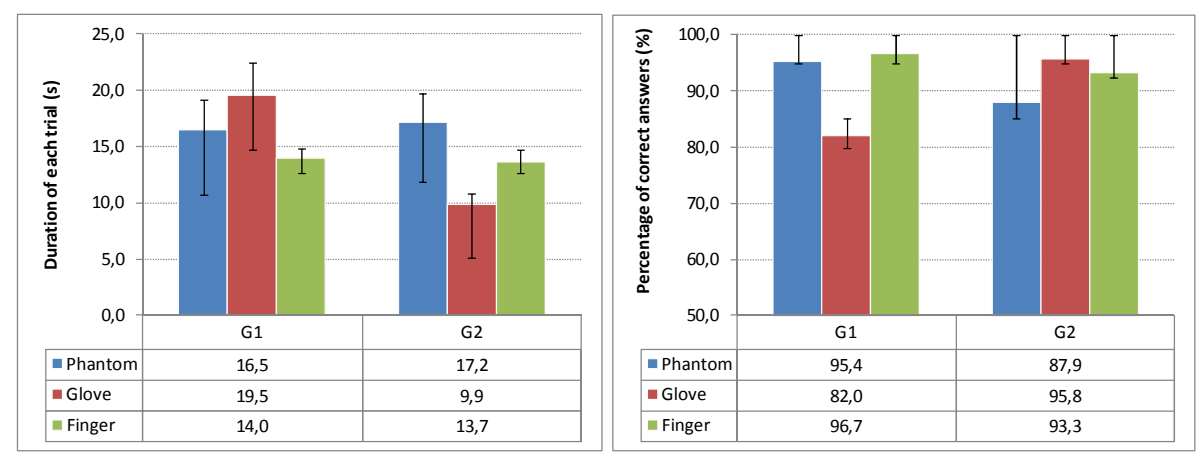

Fig. 2. Average duration of trials (left) and percentage of correct answers (right). Bounded lines represent the interval between the first and third quartile of the samples.

\section{Results and Discussion}

Fig. 2 shows both the average duration of each attempt (left), and the average percentage of correct answers (right). As expected for the first group of textures, the method of direct tactile stimulation is the fastest and the one which provides the higher percentage of correct answers, due to the advantage of having the entire surface of the fingertip to follow contours and identify shapes. Similarly, the force feedback percentage of hits is very close, because the device guides the user's finger when the cursor passes over an area of depression, reducing the cognitive effort. In the case of the dataglove, the stimulation is performed in one point and does not allow accurate tracking of the border. In this case, the user is required to develop a detection strategy different from the one followed naturally. This requires the user to make a greater effort, resulting in higher error rate and time consumption.

For the second set of textures, the most efficient method in terms of both time and hit rate is the vibrotactile feedback, even improving the method of direct stimulation. To discriminate between different patterns, the user typically scans the texture across the lines at constant speed, trying to identify the timing or frequency of the marks. That is why the vibration is appropriate, since the user perceives clearly the necessary information. In contrast, when the user swipes its finger across the paper textures much more spatial information is received that has to be discarded, so the effectiveness is not as good in terms of time and error rate. Finally, the separation between the lines is perceived mostly at a kinaesthetic level when using the force feedback, affecting the performance of this feedback method in this test. 


\section{Conclusions}

A texture discrimination experiment has been conducted in order to compare force and tactile feedback with the vibration feedback of a glove developed at our laboratory. Vibrotactile feedback seems to be the most effective method to distinguish between texture patterns that can be identified by the frequency changes of their surface features while rubbing it with the finger. However, in tasks where a precise spatial recognition is needed to identify shapes it has not resulted as effective as other methods, yet has proved to be useful.

This study shows some interesting results that can be corroborated in a future work by expanding both the number of users and the variety of textures to detect, also recognizing textures or shapes in the space, where the use of multiple vibrotactile actuators can be an advantage compared to the localized force feedback.

Acknowledgments. This work has been supported by the projects PEII09-0054-9581 and TIN2008-06596-C02-01. Thanks to the users who have participated voluntarily in the experiments.

\section{References}

1. Robles-De-La-Torre, G.: The Importance of the Sense of Touch in Virtual and Real Environments. IEEE Multimedia, 24-30 (2006)

2. Minsky, M., Ming, O., Steele, O., Brooks Jr, F.P., Behensky, M.: Feeling and seeing: issues in force display. In: ACM Symposium on Interactive 3D graphics, pp. 235-241 (1990)

3. Kuchenbecker, K., Fiene, J., Niemeyer, G.: Improving contact realism through event-based haptic feedback. IEEE Transactions on Visualization (2006)

4. Okamura, A.K., Dennerlein, J., Howe, R.D.: Vibration feedback models for virtual environments. In: IEEE Int. Conf. on Telerobotics and Automation, pp. 1-6 (1998)

5. Hollins, M., Bensmaïa, S., Roy, E.: Vibrotaction and texture perception. Behavioural Brain Research 135, 51-56 (2002)

6. Wellman, P., Howe, R.D.: Towards realistic vibrotactile display in virtual environments. In: Symp. on Haptic Interfaces for Virtual Env. and Teleoperator Sys. (1995)

7. Gurari, N., Smith, K., Madhav, M., Okamura, A.M.: Environment discrimination with vibration feedback to the foot, arm, and fingertip. In: IEEE Int. Conf. on Rehabilitation Robotics, pp. 343-348 (June 2009)

8. Nintendo Rumble Pak, http://www.nintendo.es/NOE/es_ES/systems/accesorios_1225.html

9. CyberGlove Systems, http: / / www . cyberglovesystems . com/

10. Kyung, K.U., Lee, J.Y., Park, J.S.: Comparison of force, tactile and vibrotactile feedback for texture representation using a combined haptic feedback interface. In: Oakley, I., Brewster, S. (eds.) HAID 2007. LNCS, vol. 4813, pp. 34-43. Springer, Heidelberg (2007)

11. Johansson, R.S.: Tactile Sensibility in the human hand: receptive field characteristics of mechanoreceptive units in the glabrous skin area. Journal of Physiol. 281 (1978)

12. Sensable, http: / / www . sensable.com

13. H3D Open Source Haptics, http : / / www . h3 dapi . org

14. Martínez, J., Molina, J.P., García, A.S., Martínez, D., González, P.: Desarrollo de un Guante de Datos con Retorno Háptico Vibro-táctil Basado en Arduino. JoReVir, 1-10 (2009) 